\title{
Configurando o Google Contas Inativas: percurso cognitivo e outras trilhas avaliativas
}

\author{
Maria Fernanda Lira ${ }^{1}$, Cristiano Maciel ${ }^{12}$, Daniele Trevisan ${ }^{2}$, Sílvia Amélia Bim ${ }^{3}$ \\ ${ }^{1}$ Instituto de Computação - Universidade Federal de Mato Grosso - UFMT \\ Cuiabá-MT-Brasil \\ ${ }^{2}$ Programa de Pós-Graduação em Educação - Universidade de Mato Grosso (UFMT) \\ Cuiabá-MT-Brasil \\ ${ }^{3}$ Departamento Acadêmico de Informática - UTFPR-CT \\ Curitiba-PR- Brasil \\ \{nanda.lira57@gmail.com, crismac@gmail.com, daniele.tr@hotmail.com, \\ sabimeutfpr.edu.br\}
}

\begin{abstract}
A large number of digital goods and assets are produced daily in various spaces on the internet. After users die, these goods and assets constitute their digital legacy and need a destination. Today, many systems are looking for solutions to manage post-mortem digital legacy. This study aims to evaluate the interactive system of Google Inactive Account Manager (IAM) or Inactive Account, through the mPC inspection method (Cognitive Pathway method) and compare the results of this evaluation with others provided in the literature. The results show that evaluation methods in $\mathrm{mPC}$ also contribute to improve user interaction with the system and point out issues that need improvement.
\end{abstract}

Resumo. Uma grande quantidade de bens e ativos digitais são produzidos diariamente nos diversos espaços na internet. Após a morte dos usuários, esses bens e ativos constituem o seu legado digital e precisam de um destino. Atualmente, muitos sistemas têm buscado soluções para o gerenciamento de legado digital pós-morte. Este estudo tem como objetivo avaliar o sistema interativo do Google Inactive Account Manager (IAM) ou Contas Inativas, por meio do método de inspeção mPC (método de Percurso Cognitivo) e comparar os resultados dessa avaliação com outros fornecidos na literatura. Os resultados evidenciam que os métodos de avaliação em mPC também contribuem para melhorar a interação do usuário com o sistema e apontar questões que precisam ser aprimoradas.

\section{Introdução}

Uma grande quantidade de artefatos digitais são produzidos e socializados diariamente. Esses dados continuam na rede após a morte do usuário, constituindo assim, o legado digital destas pessoas. Segundo Maciel e Pereira (2014), as pessoas têm percepções diferentes sobre o que querem que aconteça com seus bens digitais quando morrerem, por exemplo: se desejam que suas contas sejam excluídas; se desejam que apenas amigos e familiares tenham acesso a suas redes sociais, como Youtube, Facebook, Instagram etc. Entretanto, independente da vontade, é necessário expressá-la em vida.

Os SGLDs (Sistemas de Gerenciamento de Legado Digital) permitem que usuários planejem sobre o que querem que aconteça com seus bens digitais depois que falecerem. Yamauchi et al. (2021) realizaram uma pesquisa analisando SGLD sob o ponto de vista teórico, sistêmico e dos usuários. Eles tipificam os sistemas em dedicados (SDGLD) e integrados 
(SIGLD). Entre os sistemas para esta finalidade, o Google lançou em 2013 o Google Inactive Account Manager (IAM), ou Google Contas Inativas. Por meio dele, é possível que usuários definam com antecedência sobre o que querem que aconteça com seus bens digitais (Google, 2021). O Google IAM é um sistema integrado, ou seja, mesmo que não tenha como objetivo primário a destinação de bens digitais, ele tem funcionalidades para que usuários decidam com antecedência o que querem que aconteça com seus artefatos digitais.

Existem alguns estudos na literatura, relatados neste artigo, que tratam do Google IAM. Entre eles, no campo da IHC, estão o estudo de funcionalidades com engenheiros de software (Maciel, Pereira e Sztern; 2015) e com a inspeção semiótica (Prates, Rosson e Souza, 2015; 2016). Todavia, percebe-se a necessidade de uma avaliação do percurso que os usuários seguem ao utilizar a ferramenta Google IAM.

O percurso cognitivo é um método de avaliação IHC, cujo objetivo principal é avaliar a facilidade de aprendizado de um sistema interativo através da exploração em sua interface. Além disso, o avaliador se coloca no lugar do usuário e verifica se há algum problema e tenta prever se os utilizadores do sistema terão alguma dificuldade com a interação do sistema (Wharton et al., 1994).

Neste sentido, este estudo tem por objetivo avaliar o sistema interativo do Google Inactive Account Manager, por meio do método de inspeção mPC (método de Percurso Cognitivo). Adicionalmente, resultados desta avaliação são comparados com outros fornecidos na literatura. Para tal, desenhou-se uma pesquisa do tipo exploratória, com abordagem qualitativa.

Na Seção 2 será apresentado o referencial teórico com trabalhos que abordam temas relacionados a esta pesquisa. Na Seção 3, será apresentada a metodologia utilizada neste estudo. $\mathrm{Na}$ Seção 4, por sua vez, será apresentado o método de inspeção do percurso cognitivo, aplicado na ferramenta do Google IAM. Por fim, apresentaremos a conclusão com os resultados obtidos nesta pesquisa.

\section{Referencial Teórico}

Para o desenvolvimento desta pesquisa, além de conceitos gerais necessários para compreensão da temática em estudo, foi realizada uma revisão bibliográfica com pesquisas de autores brasileiros que ressaltam a importância do sistema Google IAM, o qual permite o gerenciamento de legado digital. Além disso, serão abordados trabalhos que utilizam o método de percurso cognitivo.

De acordo com Carroll e Romano (2011), legado digital pode ser definido como um conjunto de itens digitais, como: fotos, vídeos, arquivos, livros digitais, e-mails, contas em redes sociais, entre outros materiais digitais que são acumulados por indivíduos ao longo do tempo. Para destinação do legado, Google IAM permite duas possibilidades aos usuários, sendo elas: 1) a exclusão total da conta após o falecimento do proprietário de conta; e 2) que os usuários registrem até dez contatos herdeiros, para que os mesmos recebam os artefatos digitais. Além disso, o proprietário da conta pode deixar uma mensagem de despedida a seus herdeiros, o mesmo também pode escolher o que cada herdeiro irá receber e o proprietário de conta pode a qualquer momento excluir ou adicionar um novo herdeiro (Google, 2021).

Logo, quando a ferramenta percebe a inatividade da conta, sendo o tempo de inatividade pré-definido por cada titular da mesma, o sistema verifica a morte do usuário e, assim que a mesma for confirmada, o contato de confiança, informado previamente pelo usuário, deve baixar todos os dados e seguir as instruções que o titular da conta deixou Maciel e Pereira (2013). Cabe salientar que, entre as soluções existentes no mercado, o Google IAM é 
uma das que disponibiliza funcionalidades de gerenciamento do legado a sistemas de forma integrada a um sistema maior, não sendo um sistema dedicado ao tratamento de legados em si (Maciel, 2021).

Algumas pesquisas, no campo de IHC, têm pesquisado e avaliado este gerenciador. No primeiro livro de IHC no campo do legado (Maciel e Pereira, 2013), ao longo da obra, os autores citam e comentam o Google IAM, pelas possibilidades que ele começou a oferecer no tratamento do legado. Ressalta-se que notícias apontam o lançamento desta funcionalidade no ano de 2013 (Robertson, 2013). No livro do mesmo ano, as análises realizadas por autores são com relação à visão de engenheiros de software sobre essas funcionalidades.

Maciel, Pereira e Sztern (2015) questionam em que medida as questões técnicas e culturais evidenciadas por engenheiros de software e projetistas da interação, confrontados com o Google Inactive Account, podem subsidiar a discussão de dispositivos legais relacionados ao legado digital. Assim, levantam questões legais que emergem a partir da disponibilização em aplicações de possibilidades para configuração do legado digital pós-morte, sob a ótica dos engenheiros. Com base nos achados, os autores concluem, entre outros, a fragilidade dos dispositivos legais que amparam questões como direito sucessório, direito ao esquecimento, temporalidade das informações de contato e impasses técnicos.

Prates Rosson e Souza (2015) exploraram o conceito de antecipação da interação, ou seja, a relação com as consequências futuras das ações tomadas por um usuário em um sistema. Para projetar sistemas que envolvem interações com efeitos futuros, as autoras propõem que projetistas de sistemas considerem os seguintes desafios: suporte à antecipação, representação, custos x benefícios, negociação e mitigação de conflitos, e definição de valores padrão. Para realizar os estudos, foi analisado o Google Inactive Account usando o Método de Inspeção Semiótica (SIM).

Já Prates, Rosson e Souza (2016), em avanço à pesquisa anterior, utilizaram o SIM em combinação com os desafios configuráveis de antecipação de interação (CIAC). Os resultados obtidos no estudo mostram que o Google IAM não oferece suporte para que os usuários explorem os efeitos futuros de suas configurações; não há informações com relação ao limite de tempo para que contatos confiáveis façam download de dados; e aos usuários que desejam excluir a conta permanentemente e tenham publicações em redes sociais do Google (por exemplo, YouTube), não é informado pelo sistema sobre o que aconteceria com essas publicações.

Pereira (2017) realizou estudos utilizando o método de inspeção semiótica. O trabalho analisou diferentes ferramentas que permitem a antecipação da interação e outras dimensões relevantes para a representação e gerenciamento do legado digital. O estudo cita o Google Inactive Account, todavia este não é objeto de avaliação.

Considerando os estudos já realizados, percebe-se que o método de inspeção mPC (método de Percurso Cognitivo) ainda não foi utilizado para inspeção do Google IAM. Marques et. al. (2015) realizaram um estudo analisando a usabilidade de sítios governamentais. Os autores compararam diferentes métodos de IHC, entre os quais está o método de percurso cognitivo. Com base nas avaliações, os autores sugeriram melhorias para estes sistemas e reforçaram a necessidade de uso de múltiplos métodos para avaliação de sistemas. Por sua vez, Bim, Salgado e Leitão (2016) realizaram um estudo fazendo uma análise comparativa em três métodos de inspeção, são eles: Avaliação Heurística, Percurso Cognitivo e Método de Inspeção Semiótica. O objetivo foi esclarecer e orientar sobre o aprimoramento de metodologias para IHC. Os resultados do estudo apontam que o mPC mostra-se potencialmente útil para a 
pesquisa de aspectos cognitivos e que o SIM oferece recursos para questões relativas à comunicabilidade.

\section{Metodologia}

A pesquisa em tela é do tipo exploratória, com abordagem qualitativa. Inicialmente, face à carência de estudos apontadas na literatura, partiu-se para a análise da facilidade de configuração do sistema Google Inactive Account Manager, por meio do método de percurso cognitivo (mPC). O mPC é um método no qual o avaliador percorre a interface inspecionando as ações projetadas para um usuário concluir cada tarefa utilizando o sistema (Barbosa e Silva, 2010).

O método possui uma sequência de ações que seriam os passos de um percurso bem sucedido com vistas a atingir o objetivo final do usuário. A ação no caminho do sucesso é definida pelo avaliador, o mesmo tenta se colocar no papel do usuário e detalha como seria sua interação com o sistema naquele momento. Para avaliação do sistema, são respondidas 4 perguntas pré definidas no mPC, a saber: 1) o usuário tentará alcançar o resultado correto?; 2) o usuário consegue notar que a ação correta está disponível?; 3) o usuário consegue associar as ações disponíveis com o seu objetivo final?; 4) o usuário vai perceber que foi feito um progresso na direção do seu objetivo? (Barbosa e Silva, 2010)

Neste experimento, o método do percurso cognitivo foi realizado por uma pesquisadora que tem conhecimentos em IHC (Interação Humano-Computador). A pesquisadora já utilizou o sistema e além disso, já realizou teste com usuários com esse sistema (Google IAM), todavia nunca havia realizado o percurso cognitivo deste sistema.

Após essa etapa, para discussão sobre o sistema, foram compilados achados nas avaliações dos trabalhos relacionados identificados no referencial teórico, possibilitando uma análise mais ampla do objeto. Tais resultados serão apresentados na próxima Seção.

Cabe salientar que está pesquisa faz parte do projeto de pesquisa DAVI (Dados Além da Vida) ${ }^{1}$, do Instituto de Computação da Computação da UFMT, cujo objetivo principal é realizar pesquisas sobre morte e tecnologias, buscando conscientizar usuários sobre a destinação de bens digitais, em suas múltiplas formas.

\section{Resultados}

Nesta Seção, apresentamos a análise sistemática utilizando o mPC (método de Percurso Cognitivo) no sistema Google IAM. A seguir, são detalhadas as etapas seguidas.

\subsection{Resultados obtidos pelo método de mPC}

Para realizar a análise deste método, é necessário incluir a descrição do usuário, cenário e os passos corretos que devem ser realizados para chegar no objetivo final, respondendo as perguntas definidas pelo $\mathrm{mPC}$, como descrito na metodologia.

Em relação ao perfil do usuário, podemos ressaltar que são usuários em geral, de todas as idades, que fazem uso dos serviços Google, como por exemplo: Gmail, Dropbox, Youtube, Docs etc., e que desejam definir um futuro para seu legado digital.

O cenário da tarefa foi o Google que possui o gerenciador de contas inativas, no qual usuários podem destinar seus bens digitais para herdeiros, sendo esses bens diversos.

\footnotetext{
${ }^{1}$ http://lavi.ic.ufmt.br/davi/
} 
Para a aplicação do mPC, a pesquisadora avaliadora se colocou no contexto de utilização do sistema para que fosse possível responder às questões propostas pelo método. A sequência correta de ações para configuração do Google Inactive Account são:

1. "Fazer login"

2. Clicar em "Privacidade e Personalização", ou, "Dados de Personalização".

3. Criar um plano para sua conta.

4. Clicar em iniciar e definir quando sua conta do Google deve ser considerada inativa.

5. Escolher quem o sistema deve notificar e o que compartilhar.

6. Decidir se sua Conta do Google inativa deve ser excluída.

7. Confirmar o Plano.

A pesquisadora, durante o mPC do sistema (IAM), cumpriu as ações 1 e 2 de forma tranquila e sem dificuldades, tendo em vista que já são ações realizadas na utilização de todos os aplicativos Google e para definições de privacidade e personalização de todas as opções do sistema. A ação 3 consistia em criar um plano para sua conta (como mostrado na Figura 1), essa opção pode causar uma grande dificuldade para usuários que não tenham habilidade em manusear a ferramenta. O usuário poderia se confundir e não achar a opção "Criar um plano para sua conta", pois o mesmo teria que procurar tal opção e, por conta disso, poderia desistir de configurar a sua conta, não definindo um futuro para seu legado digital.

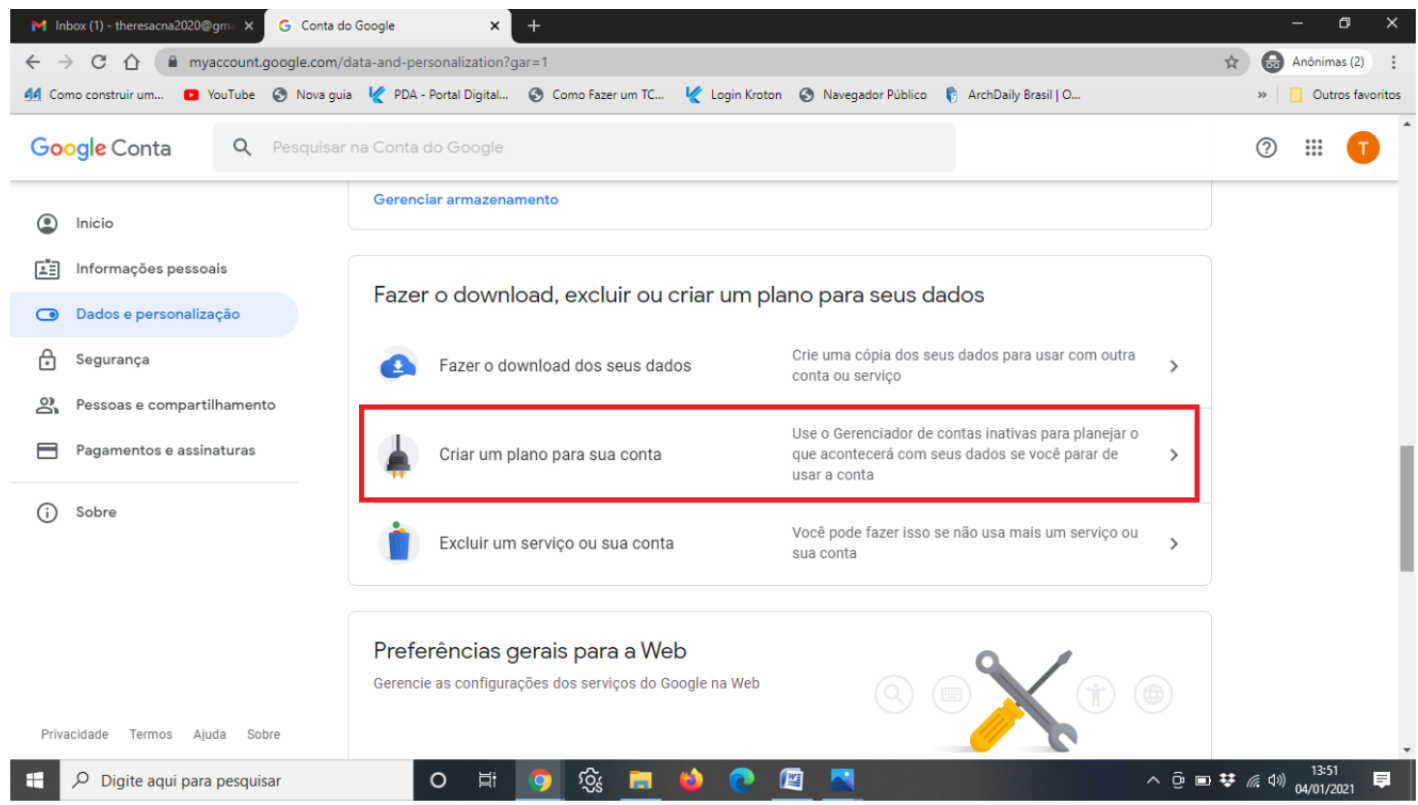

Figura 1. Criar um plano para sua conta

A ação 4 também não gera grandes dificuldades e é de fácil definição. Já na ação 5, o sistema poderia falhar pois essa etapa deixa subentendido que usuários devem obrigatoriamente "adicionar pessoa", sendo que talvez o usuário deseja apenas excluir a sua conta e isso não está especificado. Pela falta de informação, o usuário poderá "adicionar pessoa" sendo que basta o usuário clicar em "próximo" até chegar na opção "exclusão de conta". Nas ações 6 e 7, os usuários do sistema conseguiriam realizar tais ações tranquilamente.

\subsection{Discussões}


Na tabela 1, são apresentados três trabalhos encontrados, que utilizam distintos métodos de IHC para avaliação do Google IAM, ordenados por ano de publicação.

Tabela 1. Avaliações do Google IAM a partir de diferentes métodos de inspeções

\begin{tabular}{|c|c|c|}
\hline Autores & Método(s) & Principais achados das avaliações \\
\hline $\begin{array}{l}\text { Maciel, } \\
\text { Pereira e } \\
\text { Sztern } \\
(2015)\end{array}$ & $\begin{array}{l}\text { Survey com } \\
\text { usuários }\end{array}$ & $\begin{array}{l}\text { - Necessidade de considerar os valores humanos quando se } \\
\text { trata de herança (privacidade, confiança, ética e conflitos } \\
\text { interpessoais) } \\
\text { - As questões legais precisam ser discutidas (direito sucessório } \\
\text { e direito ao esquecimento) } \\
\text { - No que diz respeito a volição, o sistema utiliza a expressão } \\
\text { "conta inativa" ao invés de "morte", para ganhar maior adesão } \\
\text { - Herdeiro não é consultado antes da destruição dos dados e } \\
\text { não é orientado sobre o que deve fazer } \\
\text { - Necessidade de compatibilizar o direito do herdeiro de } \\
\text { escolher receber ou não um bem digital } \\
\text { - Devido à imprevisibilidade da morte, o usuário mantém } \\
\text { informações que não gostaria de compartilhar } \\
\text { - Caso o herdeiro indicado pelo usuário altere endereço, e-mail } \\
\text { e/ou telefone não será possível realizar o contato } \\
\text { - Se o usuário por motivos de saúde ou de reclusão não acessar } \\
\text { a conta pelo período indicado, pode haver a inativação sem ele } \\
\text { de fato ter falecido. O usuário também pode errar na } \\
\text { configuração do limite de tempo }\end{array}$ \\
\hline $\begin{array}{l}\text { Prates, } \\
\text { Rosson e } \\
\text { Souza } \\
(2015)\end{array}$ & $\begin{array}{l}\text { Método de } \\
\text { inspeção } \\
\text { semiótica } \\
\text { (SIM) }\end{array}$ & $\begin{array}{l}\text { - Quanto ao suporte de antecipação, na página de ajuda existe } \\
\text { uma explicação muito breve sobre o que significa ter seus } \\
\text { dados deletados } \\
\text { - O Google IAM não fornece meios aos usuários para } \\
\text { visualizar (ou experimentar) impactos que resultarão de sua } \\
\text { configuração } \\
\text { - Em relação aos custos x benefícios, embora seja de fácil } \\
\text { configuração, ressalta-se os custos emocionais, que podem } \\
\text { resultar da morte do usuário } \\
\text { - Um contato confiável pode receber a mensagem de herdeiro } \\
\text { mas não tem mais acesso ao número do celular cadastrado e } \\
\text { isso impediria o acesso aos dados }\end{array}$ \\
\hline $\begin{array}{l}\text { Prates, } \\
\text { Rosson e } \\
\text { Souza } \\
(2016)\end{array}$ & $\begin{array}{l}\text { Método de } \\
\text { inspeção } \\
\text { semiótica } \\
\text { (SIM) em } \\
\text { combinação } \\
\text { com os } \\
\text { desafios } \\
\text { configuráveis } \\
\text { de antecipação } \\
\text { de interação } \\
\text { (CIAC) }\end{array}$ & $\begin{array}{l}\text { - A morte é detectada apenas pela inatividade da conta, pois o } \\
\text { sistema só perceberá que o usuário morreu pelo tempo de } \\
\text { inatividade do mesmo } \\
\text { - Limite de tempo que a ferramenta dá aos contatos confiáveis } \\
\text { para que os mesmos façam download dos dados deixados pelo } \\
\text { proprietário da conta } \\
\text { - O Google IAM não oferece suporte para que os usuários } \\
\text { explorem os efeitos futuros de suas escolhas feitas nas } \\
\text { configurações }\end{array}$ \\
\hline
\end{tabular}


Nesta pesquisa, com o uso do Percurso Cognitivo, buscamos apresentar possíveis dificuldades que usuários podem encontrar ao tentar configurar o sistema IAM, tendo em vista que este é um fator fundamental para a utilização do sistema. Percebe-se pelos achados da Seção 4.1 que se o usuário não tivesse tanta habilidade em mexer na ferramenta, o sistema falharia na $3^{\circ}$ ação e, devido a dificuldade que o usuário pudesse encontrar, o mesmo poderia desistir de chegar até seu objetivo final. Uma solução para este problema seria inserir a opção "Crie um plano de conta" na página inicial do Google IAM, assim o usuário seria direcionado para a parte das configurações.

Todos os métodos mencionados, questionários com usuários e/ou desenvolvedores de sistemas e métodos de inspeção semiótica trouxeram significativas contribuições para avaliação em IHC, referentes a usabilidade e comunicabilidade do sistema Google IAM. Os resultados são fundamentais no que se refere à privacidade, confiança, ética, comunicabilidade, entre outros aspectos do sistema.

\section{Considerações Finais}

O objetivo trilhado para esta pesquisa foi o de avaliar o sistema interativo do Google Inactive Account Manager, por meio do método de inspeção mPC (método de Percurso Cognitivo) e comparar os resultados com outros fornecidos na literatura. O Google IAM é um sistema que trata de diferentes dados dos usuários, desde e-mails até redes sociais.

Percebe-se que diferentes métodos têm oferecido aos projetistas diferentes pontos para melhoria do sistema. Assim, reforçamos a necessidade e a importância de serem utilizados diferentes métodos de avaliação IHC, de maneira a identificar as diferentes possibilidades de usabilidade e comunicabilidade dentro do sistema. Além disso, podemos analisar que os trabalhos abordados nesta pesquisa, incluindo a pesquisa em tela, apresentam possíveis problemas à ferramenta seja na parte da execução de tarefa (como mostra neste trabalho); seja pela falta de dispositivos legais, como apontado por Maciel, Pereira e Sztern (2015); ou seja pela falta de suporte, tempo e inatividade do sistema (IAM) como relatado por Prates, Rosson e Souza (2017).

Assim, como trabalhos futuros, seria interessante realizar um estudo com usuários de diferentes faixas etárias, que utilizem o sistema interativo Google Inactive Account, com o objetivo de testar a facilidade de uso e comunicabilidade do sistema e propor melhores soluções para o sistema via prototipação. Ainda, a busca por pesquisas realizadas em outros países pode gerar dados interessantes, todavia é importante reforçar que a avaliação das interfaces em língua portuguesa e as leis de cada país podem refletir em mudanças do Google IAM ao redor do mundo.

\section{Referências}

Barbosa, S. D. J. and Silva, B.S. (2010). Interação Humano-Computador. Elsevier.

Bim, S. A., Salgado, L. C. C. and Leitão, C. F. (2016) "Evaluation by inspection: Comparing methods of practical, cognitive and semiotic basis." Proceedings of the 15th Brazilian Symposium on Human Factors in Computing Systems. 2016.

Carroll, E. and Romano, J. (2011) Your Digital Afterlife: When Facebook, Flickr and Twitter Are Your Estate. What's Your Legacy, New Riders.

Google. (2021) Google Account, 2021 <https://www.google.com/account/about/> 
Maciel, C. and Pereira, V. C. (2014) A morte como parte da vida digital: uma agenda de pesquisa em IHC. In: Proceedings of the 13th Brazilian Symposium on Human Factors in Computing Systems. p. 441-444.

Maciel, C. and Pereira, V. C.. (2013) Digital Legacy and Interaction: Post-Mortem Issues. Switzerland: Springer, 2013. 144p.

Maciel, C. (2021) Sistemas Gerenciadores de Legado Digital: implicações tecnológicas e legais em redes sociais. In: Brazilian Workshop on social Network analysis and mining (Brasnam), 10. , 2021, Evento Online. Anais [...]. Porto Alegre: Sociedade Brasileira de Computação, p. 275-286. ISSN 2595-6094. DOI: https://doi.org/10.5753/brasnam.2021.16148.

Maciel C., Pereira V.C., Sztern M. (2015) Internet Users' Legal and Technical Perspectives on Digital Legacy Management for Post-mortem Interaction. In: Yamamoto S. (eds) Human Interface and the Management of Information. Information and Knowledge Design. HIMI 2015. Lecture Notes in Computer Science, vol 9172. Springer, Cham. https://doi.org/10.1007/978-3-319-20612-7_59

Marques, V. et al. Inspeção da Interação em sítios governamentais: uma comparação entre métodos. In: Anais do XI Simpósio Brasileiro de Sistemas de Informação. SBC, 2015. p. 647-654.

Pereira, F. H. S. (2017). Investigação sobre antecipação dos impactos da interação em sistemas de gerência de legado digital de usuários.

Prates, R. O.; Rosson, M. B. and Souza, C. S. (2017) Analyzing the Communicability of Configuration Decision Space Over Time in Collaborative Systems through a Case Study. SBC Journal on Interactive Systems, v. 8, n. 2, p. 62-76, 2017.

Prates, R. O.; Rosson, M. B. and Souza, C. S. (2015) Making decisions about digital legacy with Google's inactive account manager. In Abascal, J.; Barbosa, S.; Fetter, M.; Gross, T.; Palanque, P. \& Winckler, M.(Eds.) Human-Computer Interaction -- INTERACT 2015, Springer International Publishing, 2015, 9296 , pp. 201-209

Prates, R. O.; Rosson, M. B. and Souza, C. S. (2015). Interaction Anticipation: Communicating Impacts of Groupware Configuration Settings to Users. In: International Symposium on End User Development. Springer, Cham. p. 192-197.

Prates, R. O.; Rosson, M. B. and Souza, C. S. (2016) Analysis of Configuration Decision Space Over Time: The Google Inactive Manager Account Case. In Proceedings of the 15th Brazilian Symposium on Human Factors in Computing Systems (IHC '16). Association for Computing Machinery, New York, NY, USA, Article 23, 1-10. DOI:https://doi.org/10.1145/3033701.3033724

Robertson, A. (2013) Google launches 'Inactive Account Manager' to deal with your data when you die. Theverge. https://www.theverge.com/2013/4/11/4213126/google-launches inactive-account-manager-to-manage-data-after-death.

Wharton, C., Rieman, J., Lewis, C., \& Polson, P. (1994). The cognitive walkthrough method: A practitioner's guide. In Usability inspection methods (pp. 105-140).

Yamauchi, E. Maciel, C. Mendes, F. Ueda, G. and Pereira, V. C. (2021) Digital Legacy Management Systems: Theoretical, Systemic and User's Perspective. In: 23rd International Conference on Enterprise Information Systems, 2021, Online Streaming. DOI: $10.5220 / 0010449800410053$ 